\title{
Umblical metastasis in cervical cancer
}

\author{
Nadereh Behtash • Nili Mehrdad • Amir Shamshirsaz • \\ Razieh Hashemi · Farnaz Amouzegar Hashemi
}

Received: 14 December 2007 / Accepted: 26 February 2008 / Published online: 1 April 2008

(C) The Author(s) 2008

\begin{abstract}
Introduction Objective cutaneous metastasis from carcinoma of the uterine cervix is an uncommon occurrence. The outcome of patients with skin metastasis is usually poor as they are often associated with locoregional recurrence. This metastasis impairs the quality of life and shortens survival. Consequently, physicians should be aware of the possible existence of skin metastasis in cervical cancer. Clinical suspicion should lead to a careful additional evaluation whenever a cutaneous nodule presents in the course of the disease.

Case report We report a rare case of metastasis from squamous cell carcinoma of cervix to the umbilicus. The patient was diagnosed with stage IIB FIGO. Four months after chemoradiation patient presented with a nodule in the umbilical region. Biopsy performed and metastatic squamous cell carcinoma was diagnosed. Patient underwent salvage therapy with paclitaxel and carboplatin.

Conclusion To the best of our knowledge, this is a rare reported occurrence of umbilical metastasis in cervical cancer without prior laparotomy or laparoscopy. This metastatic lesion has a grave prognosis and the mean survival is about 3 months like our case.
\end{abstract}

Keywords Cervical cancer $\cdot$ Metastasis $\cdot$ Umblical

\section{Introduction}

Carcinoma of the cervix is the second most common neoplasm worldwide. Early detection and improved treatment

N. Behtash · N. Mehrdad · A. Shamshirsaz · R. Hashemi ·

F. Amouzegar Hashemi $(\bowtie)$

Vali-E-Asr Hospital, Tehran, Iran

e-mail: fahashemi@yahoo.com techniques have resulted in better control of cancer and longer survival, however, invasive and metastatic disease still occurs.

Carcinoma of the cervix recurs most commonly locoregionally after surgery and/or radiotherapy. Distant metastases are rare and lungs, bone and liver are the main sites $[1,2]$. Metastasis to the skin occurs rarely, regardless of the nature of the primary systemic neoplasm. Skin metastasis originating from cervical cancer is particularly unusual, even in the terminal stage of disease [3]. The most common site of skin metastasis in carcinoma of cervix is the anterior abdominal wall (especially at the drain site), vulva, lower extremity and anterior chest wall [3-5]. We report a case of post-radiation carcinoma of cervix stage IIB who had an umbilical metastasis which is a very rare case of cutaneous metastasis, 4 months after completion of treatment.

\section{Case report}

A 53-year-old postmenopausal woman was referred on July 2007 to the Gynecology Oncology Department, Valie-Asr Hospital, Tehran Medical University, Iran, as a postradiation case of carcinoma cervix FIGO stage IIB. She had earlier presented to the same department on December 2006 with complaint of vaginal bleeding lasted for 5 months. The pelvic examination revealed a $4-\mathrm{cm}$ cervical lesion extending to involve both parametrium. A cervical biopsy confirmed nonkeratinizing large cell squamous cell carcinoma. On investigation, biochemistry profiles and chest X-ray [postero-anterior (PA)] were within normal limits. Computed tomographies of the abdomen and pelvis were normal except parametrial involvement. No liver or other distant metastasis was found. Intravenous pyelogra- 
phy, cystoscopy and proctoscopy were within normal limits as well. She was thus staged as FIGO stage IIB. The patient was treated by external beam radiotherapy (EBR) with telecobalt equipment theratron 780 . Whole pelvis total dose was $54 \mathrm{~Gy}$ with four-field box technique employed with anterior, posterior and two lateral fields with classic limits. EBR was given concomitant with intavenous weekly cisplatin $40 \mathrm{mg} / \mathrm{m}^{2}$. Totally the patient received five platinum injections. Brachytherapy was performed 2 weeks after EBR in two sessions 1 week apart. The dose was prescribed at point A defined by Manchester system with tandem and two medium size colpostat with low dose rate/medium dose rate selectron machine. We used dose rate of $190 \mathrm{cGY} / \mathrm{h}$ and $12 \mathrm{~Gy}$ was given in each session that made the total dose of brachytherapy to point A 24 Gy with medium dose rate regimen. Radiotherapy finished on February 2007. Four months after completion of therapy, a soft tissue lesion in the region of the umbilicus developed. Local examination revealed an umbilical lesion with watery discharge (Fig. 1).

Cervical biopsy showed persistent disease. MRI of the abdomen and pelvis was performed and the only finding was a mild increasing in the thickness of the posterior wall of bladder. Abdomen computed tomography showed no liver metastasis. Chest X-ray [postero-anterior (PA)] was in normal limits. A skin biopsy performed for the patient and metastatic squamous cell carcinoma was diagnosed (Fig. 2). Salvage therapy initiated for the patient. Paclitaxel at $160 \mathrm{mg} / \mathrm{m}^{2}$ was given, followed by intravenous infusion of carboplatin dosed to an area under the curved of six (maximum dose $600 \mathrm{mg}$ ) on day 1 . She received six courses with 3 weeks interval. Following two cycle of cisplatin-based therapy, there was decrease in size of the umbilical skin lesion. On the follow-up patient died after 3 months of receiving salvage therapy.

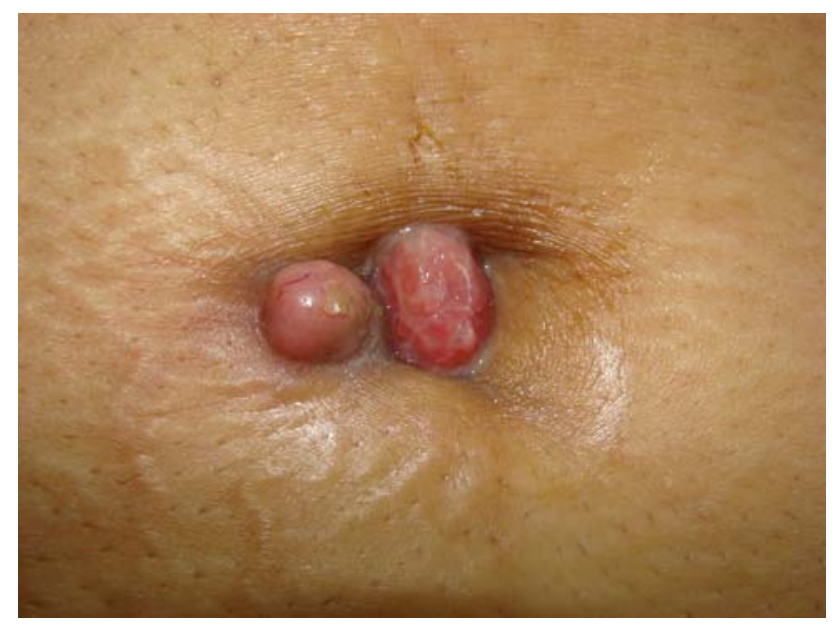

Fig. 1 Umblical metastase

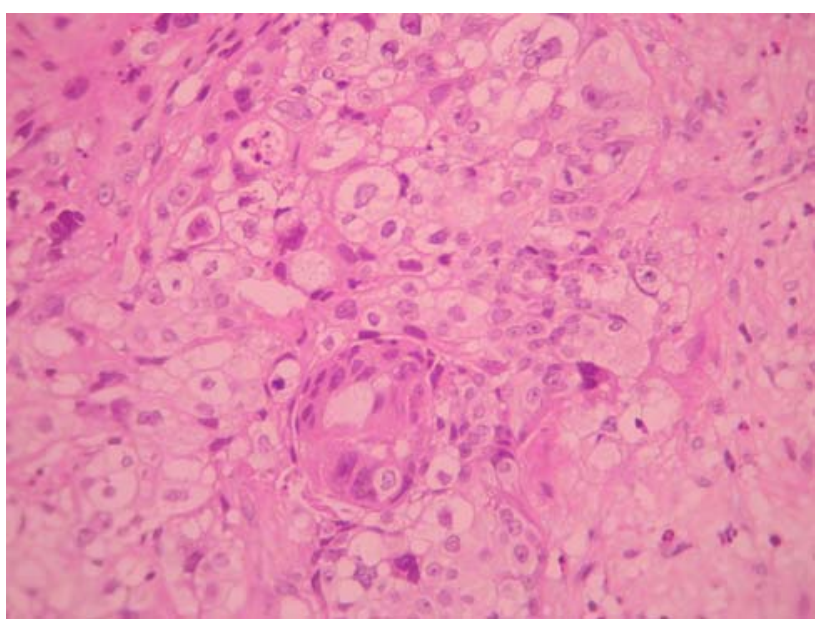

Fig. 2 Light microscopy

\section{Discussion}

The most common solid tumors metastasizing to the skin are breast, gastrointestinal, and ovarian cancers [6]. Metastasis to the skin occurs rarely in gynecologic cancer, especially in cervical carcinoma [3]. There has been no difference in the incidence of skin metastasis among the clinical stages [5]. The incidence of skin metastasis seemed to be higher in patients with adenocarcinoma and undifferentiated carcinoma than in patients with squamous cell carcinoma [7]. Our patient had stage IIB squamous cell cervical carcinoma. In uterine cervical cancer, skin metastasis has been reported in abdominal wall, vulva, anterior chest wall and lower extremity $[4,5]$. This is consistent with other carcinomas in that the metastasis to the skin is commonly located near the site of the primary tumor [8]. Skin metastases from cervical carcinoma occur predominantly in cases of tumor recurrences [3]. A review of literature shows that most of skin metastases occurred after an interval less than 21 months except three cases reported by Behtash et al. [3], Selo-Ojema et al. [9], Srivastava et al. [10] although the second case was adenocarcinoma of the cervix. In the present case, the patient developed umbilical metastasis without prior laparascopy or laparotomy. Skin involvement was observed directly, so that deduction concerning its mechanism ought to be reliable and have applicability to the general question of the mode of spread of cervical cancer. The mechanism of involvement was thought to be retrograde lymphatic spread from the corresponding regional lymph nodes [10]. This finding seemed important because it emphasized the predominant role of lymphatics in the spread of cervical carcinoma.

The prognosis associated with cutaneous metastasis of cervical carcinoma is poor. The mean reported survival of these patients is 3 months [11]. These patients are not 
amenable to curative treatment because of locally advanced or metastatic disease. The intent of treatment remains palliation either by radiation, chemotherapy, surgery alone or in combinations [5]. Cis-platinum is the single most active agent for controlling the symptoms [3], as we gave the patient carboplatin in combination with paclitaxel.

The present case is unusual in that case metastasis occurred to the normal skin (umbilical region) in squamous cell carcinoma, stage IIB 4 months after completion of the treatment and has a similar downhill course as reported in the literature

Open Access This article is distributed under the terms of the Creative Commons Attribution Noncommercial License which permits any noncommercial use, distribution, and reproduction in any medium, provided the original author(s) and source are credited.

\section{References}

1. Berek JS, Hacker NF (2000) Practical gynecologic oncology, Chapter 9. Lippincott Williams \& Wilkins, Philadelphia, pp 360380
2. Brady LW, O Neil EA, Farber SH (1977) Unusual sites of metastasis. Semin Oncol 4:59-64

3. Behtash N, Ghaemmaghami F, Yarandi F, Ardalan FA, Khanafshar N (2002) Cutaneous metastasis from carcinoma of the cervix at the drain site. Gynecol Oncol 85(1):209-211

4. Imachi M, Tsukamoto N, Kinoshita S, Nakano H (1993) Skin metastasis from carcinoma of the uterine cervix. Gynecol Oncol 48:349-354

5. Hayes AG, Berry AD (1992) Cutaneous metastasis from squamous cell carcinoma of the cervix. J Am Acad Dermatol 26:849-850

6. Lookingbill DP, Spangler M, Helm KF (1993) Cutaneous metastasis in patients with metastatic carcinoma: a retrospective study of 4,020 patients. J Am Acad Dermatol 29(2 pt 1):228-236

7. Brownstein MH, Helwing EB (1972) Patterns of cutaneous metastasis. Arch Dermatol 195:862-868

8. Reingold IM (1986) Cutaneous metastasis from internal carcinoma. Cancer 19:162-168

9. Selo-Ojema DO, Bhide M, Aggarwal VP (1998) Skin incision recurrence of adenocarcinoma of the cervix five years after radical surgery for stage IA disease. Int J Clin Pract 52:519

10. Srivastava K, Singh S, Srivastava M, Srivastava AN (2005) Incisional skin metastasis of a squamous cell cervical carcinoma 3.5 years after radical treatment. Int $\mathrm{J}$ Gynecol Cancer 15(6): 1183-1186

11. Pertzborn S, Buekers TE, Sood AK (2000) Hematogenous skin metastases from cervical cancer at primary presentation. Gynecol Oncol 76(3):416-417 